\title{
The Evolution of Base Station Antennas for Mobile Communications
}

\author{
$\begin{array}{ll}\text { C. Beckman } & \text { B. Lindmark }\end{array}$
}

\begin{abstract}
This paper gives a general overview of the design of base station antennas for mobile communications. It explains underlying theoretical and practical implementation aspects in mobile communication networks of today and the future. In the first part the fundamental parameters of a base station antenna are discussed in the context of radio network design. In particular we discuss parameters such as gain, radiation patterns, frequency bands and power handling and put them in the context of cell planning, propagation and capacity. In the final parts of the paper we give an overview of the underlying theory of diversity and MIMO systems.
\end{abstract}

\section{INTRODUCTION}

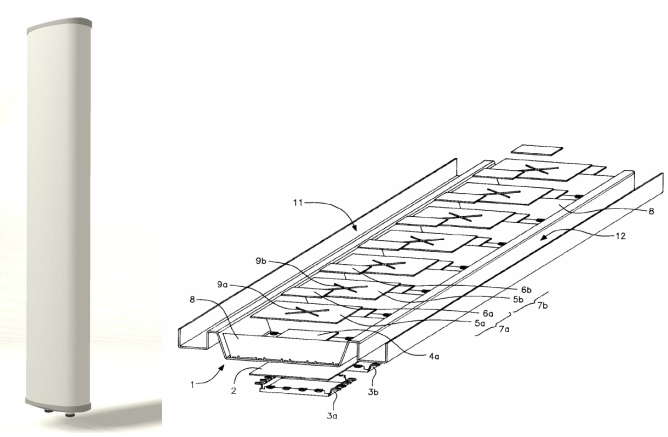

Figure 1: Dual polarized, dual band base station antenna from Powerwave [1]

Base station antennas for mobile communication systems have during the last two decades exploded in numbers in both our rural areas as well as in our city centers. These antennas (see Figure 1.) are typically 1 to 2 meters long array antennas with gains between 15 and $21 \mathrm{dBi}$ placed in towers between 25 and 75 meters above ground. They have high aperture efficiencies and are able to handle power of up to $500 \mathrm{~W}$ or more without generating either $2^{\text {nd }}$ or $3^{\text {rd }}$ order passive intermodulation products greater than the system noise floor.

In the mid 90s size reduction was an issue and manufacturers focused on designing flat panel antennas based on e.g. microstrip patches [2]. However, it was soon discovered that it was the number of antennas rather than size that was the main issue for the operators and therefore both dual polarized (for diversity) [3] and multi-band antennas [4] were soon developed.

With the introduction of digital radio systems in mobile telephony in the mid 90ties digital processing of the antenna signals also became an option. Diversity combining at the base station had been implemented already in analog systems but with the digital signal processing one could now also discuss the implementation of digital beamforming and even spatial multiplexing of signals [5].

Today diversity is almost always implemented at the base station. However, recently diversity has also been considered at the mobile terminal. The resulting "Multiple Input Multiple Output", MIMO, system is perceived as a the next important technology step in increasing the data rates that are expected to grow up to and around $100 \mathrm{Mbps}$ in e.g. the long term evolution of the $3 \mathrm{G}$ systems [6].

\section{SYSTEM ASPECTS}

\subsection{Sectorization}

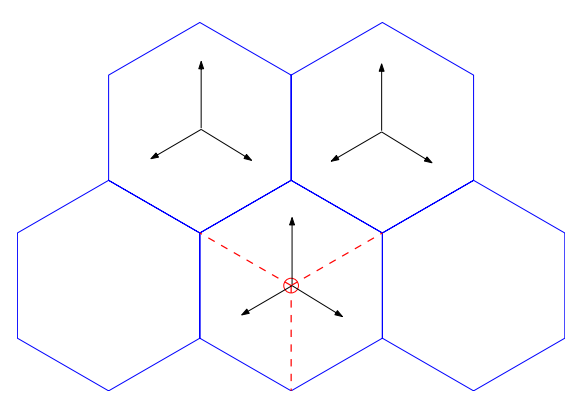

Figure 2: Rhombus cells created by replacing the omni directional antenna at the center of a hexagon by three directional antennas.

Because of the high requirements on capacity, cellular networks are most often sectorized and the use of antennas that are omni-directional in the horizontal plane is very limited today. The most popular choices are base station antennas with

\footnotetext{
${ }^{\dagger}$ Center for RF Measurement Technology, Department of Technology and Built Environment, University of Gävle, 80176 Gävle Sweden, email: cbn@hig.se, tel.: +4626648820.

\& Signal Processing, School of Electrical Engineering, Royal Institute of Technology, Stockholm, Sweden

e-mail: bjorn.lindmark@ee.kth.se, tel.: +4687906612.
} 
horizontal half power beam widths of 65 or 90 degrees. The optimum horizontal and vertical beam width is decided by the network architecture and propagation environment.

From this basic idea, the development of cellular systems has followed at least two different lines. In the U.S., systems are traditionally based on a hexagonal cellular layout with omni-directional antennas in the center of each cell. It was soon discovered that the use of directional antennas increased capacity [7], so each omni-directional antenna was replaced by three directional ones. Each directional antenna then illuminates a 120 wide sector and the original hexagon has been replaced with three rhombuses. This is known as sectorization and is seen schematically in Figure 2.

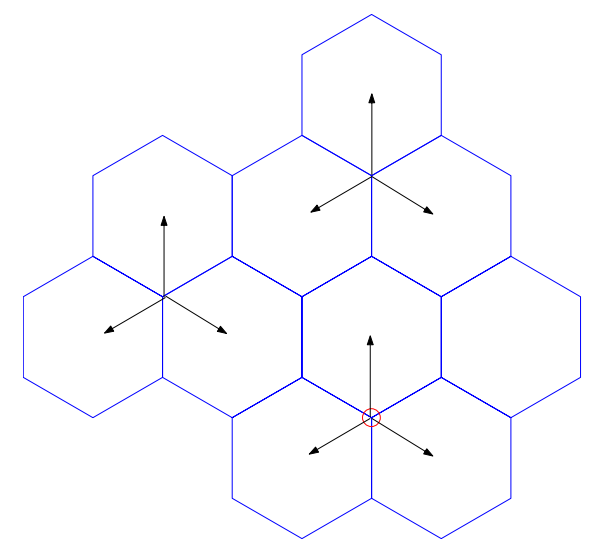

Figure 3: Hexagonal cells in a GSM system created by placing the base stations at the intersection of three hexagons

Outside the US, directional antennas are also used in hexagonal cell structure, but they are placed at the intersection of three hexagons (see Figure 3). In this case each directional antenna should cover a hexagon, rather than a rhombus. Note that although both of Figures. 2 and 3 have the base stations placed in a triangular lattice, there is a subtle but important difference: In Figure 2, the directional antennas are directed between the closest neighboring base stations and in Figure 3, they point towards a neighboring base station. This difference in the alignment of the antennas changes the cell shape from a rhombus to a hexagon. Therefore, depending on the layout of a cellular system as well as the propagation characteristics of the environment, different directional antennas are used.

\subsection{Interference}

The frequency reuse will always cause some interference and this places a constraint on how often we can re-use each radio channel. The interference can in most systems be regarded as white noise and the limiting factor is then the Signal-to-Noise-plusInterference ratio (SNIR).

$$
S N I R=\frac{S}{N+I}
$$

In GSM where the SNIR is allowed to be as low as 9 $\mathrm{dB}$, a frequency re-use factor $\mathrm{K}=9$ has been found possible.

During the early deployment of cellular systems the load on the system was low and the interference of little or no importance. With the introduction of the portable phone the operating range started to become limited by the terminal antenna gain. However, today when urban cells are often in order of a few 100 meters or less, range is not an issue any more, but now the interference limits the performance. Any means of reducing its influence on the system, e.g. by shaping the beam of the base station antenna, is therefore more than welcomed by operators as well as handset manufacturers.

Today a variety of sector antennas are offered with horizontal beam widths from about 30 to 120 degrees and gains from about 10 to $20 \mathrm{dBi}$. The use of omnidirectional antennas is very limited. The most popular choices are base station antennas with an horizontal half power beamwidth of 65 or 90 degrees.

\subsection{Choice of horizontal aperture angle}

What is then the optimum horizontal beamwidth? Let us consider a GSM cellplan as shown in Figure 3. Our objective is to provide equal signal strength on the border of the hexagonal cell. In that way the SNR is constant for all mobiles on the edge of cell, i.e. the location where communication with our base station is expected to most difficult. We assume that the received power at the mobile, $P_{R X}$, relative to the transmitted power at the base, $P_{T X}$, is described by Friis' formula [8]:

$$
P_{R X}=P_{T X}\left(\frac{\lambda}{4 \pi}\right)^{2}\left(\frac{1}{R}\right)^{\gamma} G_{R X} G_{T X}(\phi)
$$

where $G_{T X}(\phi)$ and $G_{R X}$ are the gains of the base station and terminal (omni-directional) antenna, respectively, $\lambda$ is the wavelength and $\mathrm{R}$ the distance. The azimuth angle $\phi=0$ degrees at boresight of the base station antenna. The exponent $\gamma$ has empirically [7] been found to be in the range of 3 to 5 for propagation in typical mobile communication environments.

If we approximate the hexagon shape of the cell with a circle the distance to a point on the cell border is approximately: $\mathrm{R}(\phi) \approx 2 \mathrm{r}_{0} \cos \phi$ where $\mathrm{r}_{0}$ is the length of the side of the hexagon. If we want constant radiation intensity on the cell border we get the following condition: 


$$
\frac{G_{T X}(\phi)}{R(\phi)^{\gamma}}=\frac{G_{T X}(\phi)}{\left(2 \mathrm{r}_{0} \cos \phi\right)^{\gamma}}=\text { const } .
$$

Thus, if we have $\gamma=4$, which is a typical value for a suburban environment, our ideal radiation pattern should be $\cos ^{4} \phi$. If we translate this to base station antennas, which are typically defined by their halfpower beam width (HPBW), we should look for a beam width of

$$
H P B W=2 \arccos (1 / 2)^{\gamma}=65^{\circ}
$$

If we instead assume $\gamma=2$ which could be the case for a very open flat rural terrain, we find that the optimum HPBW is instead around $90^{\circ}$.

\subsection{Vertical beam shaping.}

Through the choice of antenna length and the selection of vertical element positions and excitations in the antenna feed network, a broad range of antenna gains and vertical beam patterns can be achieved. The gain is mainly determined by the combination of the vertical and horizontal beam width but reduced by losses in the feed network!

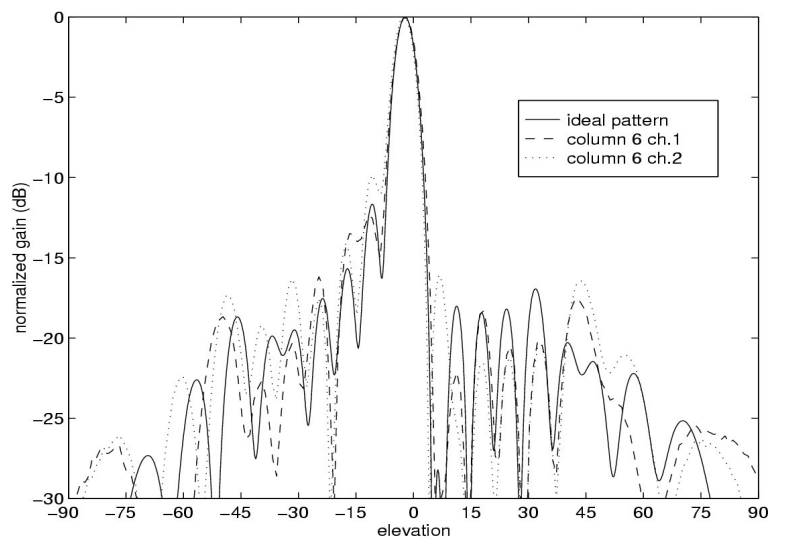

Figure 4. Vertical beam pattern of a BTS antenna (with slanted $+/$ - 45 polarization channels) for mobile communications. From [9]

If the excitation of the elements is uniform the vertical beam will form a " $\sin (x) / x$ " pattern in the far field where the half power beam width will be determined by the length of the antenna and the side lobe level will be uniform at a level of about $13 \mathrm{~dB}$ below the main beam [8].

However, in mobile telephony the capacity of each cell is limited by interference from the adjacent cells. Therefore, the antennas are used as spatial filter in order to limit this interference and improve the signal-to-noise-and-interference-ratio, SNIR. Hence, the vertical beam pattern of a base station antenna needs to be shaped in a much more complicated way than for an uniform array.

In the vertical pattern, special interest is put on (Figure 4):

- a rapid roll-off above the beam peak to a deep wide null,

- suppression of the first upper side lobe,

- slow roll-off and null fills below the beam peak

- an electrical downward beam tilt.

The rapid roll-off above the beam peak and the suppression of the first upper side lobe is to minimize interference into co-channel cells. The slow roll-off and null-fills below the beam peak ensures coverage very close to the cell site.

To create a vertical beam pattern as the one described above, the antenna design engineers makes use of the fact that the far field pattern is given by the Fourier transform of the array excitation. These excitations are then realized by the design of the feed network. Hence, the better the performance of feed network the better the antenna executes its electrical functionality. Special interest is of course often placed on the losses of the feed network since the gain is directly related to that factor. However, the phase accuracy and bandwidth of the feed network is of equally importance since the antenna is used as much as a spatial filter as a way of enhancing the SNIR.

\subsection{Electrical Tilt}
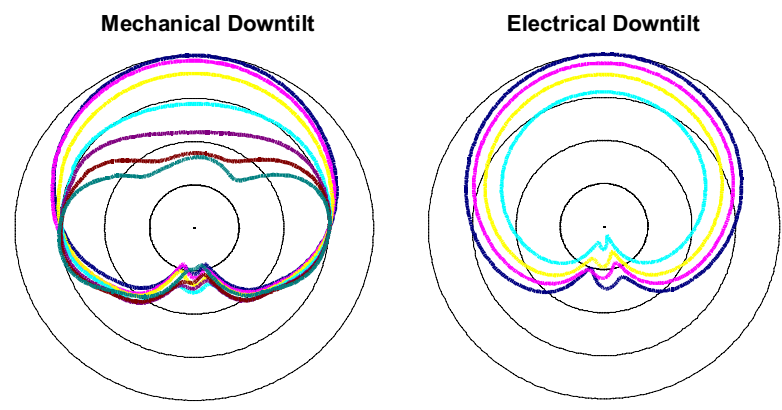

Figure 5. Illustration of the difference in foot print between Mechanical and Electrical tilt.

In order to define the coverage area of the base station, the antenna is often mechanically tilted. However, mechanical tilt will distort the footprint as seen in Figure 5.

Electrical tilt was therefore introduced since the radiation from the antenna is then directed downwards by means of the excitations in the feed network (see Figure 6). During the early roll-out of mobile networks, the operators would buy base station antennas with separate tilt for different locations. 
In order to reduce the number of antenna models, mechanically adjustable electrically tilted (MET) antennas were introduced in the mid 90-ties. The tilt could now be easily changed by mechanically changing the relative phase between the elements in the feed network using phase shifters.

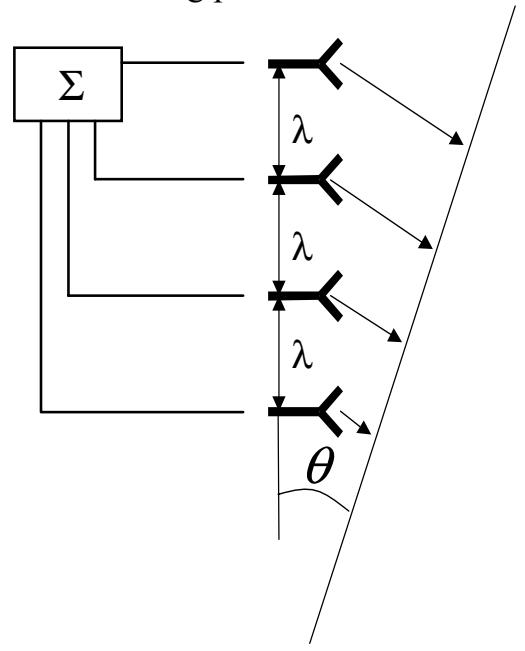

Figure 6. Illustration of Electrical tilt caused by a phase shift distributed over the excitation in the feed network.

To make the tilting even more efficient, Remote Electrical Tilt, RET, was launched in the early 2000s. The RET can tilt the antenna in real time, which can be used for changing capacity for coverage during rush hours. It is easier for fine tuning of soft handover and for changing or expanding existing networks. There are several ways to achieve the necessary phase shift, but all use electromechanical actuation. True electrical phase shift as in diode or ferrite phase shifters is in general not an option due to the very strict requirement for low intermodulation (see below). RET is currently being standardised within the $3 \mathrm{GPP}$ organisation and is today a standard functionality in many $3 \mathrm{G}$ networks.

The question is then how much electrical tilt and phase shift is necessary? If we achieve a down-tilt so that we get a null in the horizon, the interference in the adjacent cell will be very small.

Since the first null of an antenna pattern with uniform excitation falls at " $\cos \theta \approx \lambda L$ " we need to down-tilt the beam by this amount. If there are $N$ elements the spacing is approximately " $d=L / N$ ". The total phase shift across the total antenna aperture becomes $2 \pi$ regardless of length $L$. Thus, the required phase shift between the elements is rather small.

\subsection{Multiband Operations}

In the mid 90-ties the GSM $1800 \mathrm{MHz}$ band was introduced in Europe at about the same time as the PCS $1900 \mathrm{MHz}$ was opened up in North America. For the operators this meant that more spectrum and more capacity became available. However, a major problem when deploying a new cellular radio network is to find suitable site locations: Especially in urban areas, where it often is difficult to find approval for new antenna installations. In addition there is a high cost for cables, installation, rent, etc.
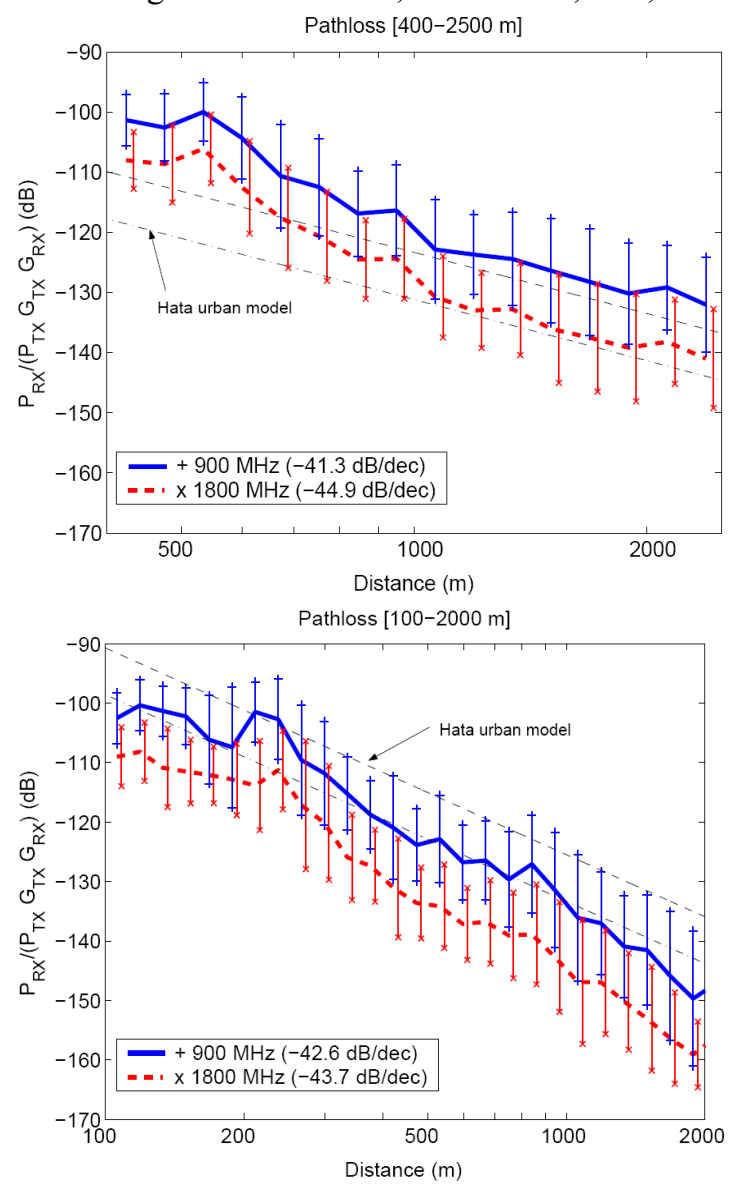

Figure 7. Measured pathloss over distance in a typical suburban (top) and urban (bottom) environment for 900 and 1800MHz. From [4].

Operators with existing GSM-900 networks therefore looked for the possibility to reuse their old sites for their new GSM1800 base stations wherever it is possible. An antenna operating on both bands was therefore an attractive solution.

When dual band antennas were first considered the question arised what characteristics they should have in each of the two frequency bands. On one hand, there is awareness that the radio link is less favorable as we move up in frequency thus making it harder to achieve the same radio coverage at $1800 \mathrm{MHz}$ as 900 $\mathrm{MHz}$ ([4] see Figure 7). This is also supported together with the fact that the attenuation increases per length unit on the $1800 \mathrm{MHz}$ band as the radio wave travels through lossy material such as concrete in buildings or tree foliage. On the other hand, there 
is a desire from many radio planners to use the same cell plan and reuse pattern for $1800 \mathrm{MHz}$ as they use for $900 \mathrm{MHz}$. This leads to two alternative antenna designs:

1. A Maximum gain antenna where the full length of the array is used for both bands.

2. An equal gain antenna where the upper frequency is tapered in order to keep the gain down to the same level as the lower frequency

For the operator it is then to decide whether he wants to achieve maximum coverage through buildings i.e. choosing design no. 1 or identical cell patterns.

\subsection{Power handling and passive intermodulation}

The average output power of a base station is typically not more than $20 \mathrm{~W}$ and the input power at the antenna connector is most often less than $10 \mathrm{~W}$. However, due to that several transmitters may be combined into each antenna and the fact that the peak power of the radio signal may be up to 10 times greater than the average, the BTS antenna is often required to withstand at least $500 \mathrm{~W}$ input power. Sometimes even $1000 \mathrm{~W}$.

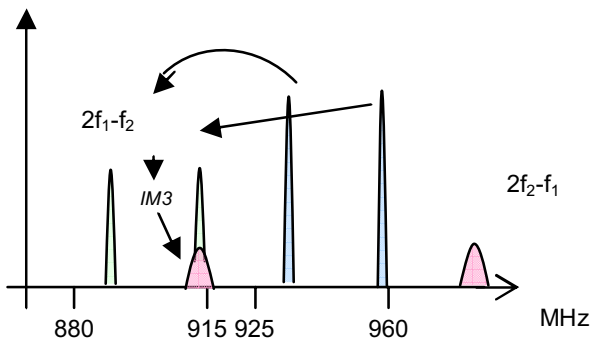

Figure 8. Illustration of the generation of third order passive intermodulation products in GSM-900.

At the same time the dynamic range of a mobile system such as GSM is very high. With a $20 \mathrm{~W}$ output power and a thermal noise of $-121 \mathrm{dBm}$ for the $200 \mathrm{kHz}$ bandwidth used, the maximum dynamic range is $164 \mathrm{~dB}$ ! Since the receive $(880-915 \mathrm{MHz})$ and transmit bands $(925-960 \mathrm{MHz})$ are quite wide, it is unfortunately possible for 3rd order intermodulation products from the transmitted signals to reach the receiver (see Figure 9) in the event that the same antenna is used both for transmitted and received signal.

In most situations this is also the case and the standard requirement on PIM for a base station antenna is normally around $150 \mathrm{dBc}$ when two sinusoidal signals each at $40 \mathrm{dBm}(10 \mathrm{~W})$ are used at input signals and the generated intermodulation products are measured at the antenna connector.
To make things worse, GSM dual band configurations become sensitive to $2^{\text {nd }}$ order intermodulation products. Such products are in the order of $\sim 10 \mathrm{~dB}$ greater than $3^{\text {rd }}$ order PIM and hence more complicated to deal with in dual band antennas.

For the UMTS band the duplex distance is so great that it is only $5^{\text {th }}$ or $7^{\text {th }}$ order products generated by the transmit signals that may generate PIM in the receive band. Hence, in theory the

\section{DIVERSITY}

\subsection{Basic theory}

The use of diversity in mobile communications to mitigate the effect of Rayleigh fading is wide-spread. It is typically used at the stationary base station, although it is quite possible to use diversity also at the terminals. For decades, the common method for base station diversity was to use spatial separation, space diversity as shown in Figure 9 below. However, since the work of Lee et. al.[1] and others in the early 70's, the interest for polarization diversity has increased and today it is the dominant base station diversity method in mobile communications. We will here summarize the theory needed for the analysis of space and polarization diversity and compare the two

The performance of a diversity system with branches 1 and 2 depends on the covariance matrix of the output signals $\mathrm{V}_{1}$ and $\mathrm{V}_{2}$ :

$$
\mathbf{C}=\left[\begin{array}{cc}
\operatorname{Var}\left\{\mathrm{V}_{1}\right\} & \operatorname{Cov}\left\{\mathrm{V}_{1}, \mathrm{~V}_{2}\right\} \\
\operatorname{Cov}\left\{\mathrm{V}_{2}, \mathrm{~V}_{1}\right\} & \operatorname{Var}\left\{\mathrm{V}_{1}\right\}
\end{array}\right]
$$

and more precisily on the eigenvalues $\lambda_{1}$ and $\lambda_{2}$ of this matrix. A general framework for antenna diversity may be obtained following the work of Collin and Zucker ([13], Eq. 4.63). The covariance of two antennas with patterns $\mathbf{E}_{1}$ and $\mathbf{E}_{2}$ may then with an approporiate normalization be written (cf. [14]:

$$
\begin{aligned}
& \operatorname{Cov}\left\{\mathrm{V}_{1}, \mathrm{~V}_{2}\right\}= \\
& =\iint_{\Omega} \operatorname{Tr}\left\{\mathbf{E}_{1}(\theta, \varphi) \mathbf{E}_{1}(\theta, \varphi)^{*} \mathbf{J}^{\prime}(\theta, \varphi)\right\} d \Omega
\end{aligned}
$$

where

$$
\mathbf{J}^{\prime}=\frac{S(\theta, \varphi)}{\chi+1}\left[\begin{array}{ll}
\chi & 0 \\
0 & 1
\end{array}\right]
$$

is a covariance matrix of the incident field. This matrix has power distribution $S$ and the polarization depends on 


$$
\chi=\frac{\mathrm{E}\left\{\left|E_{\theta}^{i}\right|^{2}\right\}}{\mathrm{E}\left\{\left|E_{\phi}^{i}\right|^{2}\right\}}
$$

the power ratio of the vertical and horizontal field components of the incident field. From the aforementioned covariance matrix, we may also calculate the complex correlation coefficient $\rho_{c}$.

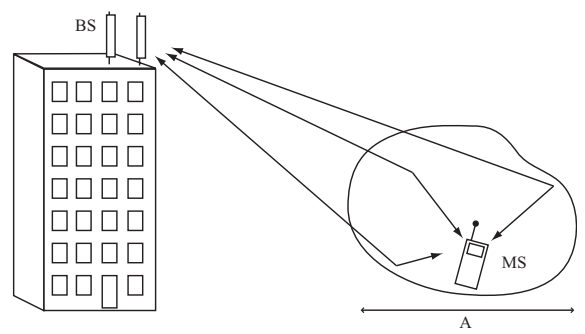

Figure 9. Illustration of a base station antenna arrangement for Space Diversity

\subsection{Space Diversity}

Since the incident field on a base station antenna is predominantly vertically polarized, space diversity antennas are typically vertically polarized. Equal average branch power is in this case obvious, but the requirements for low correlation need some further analysis.
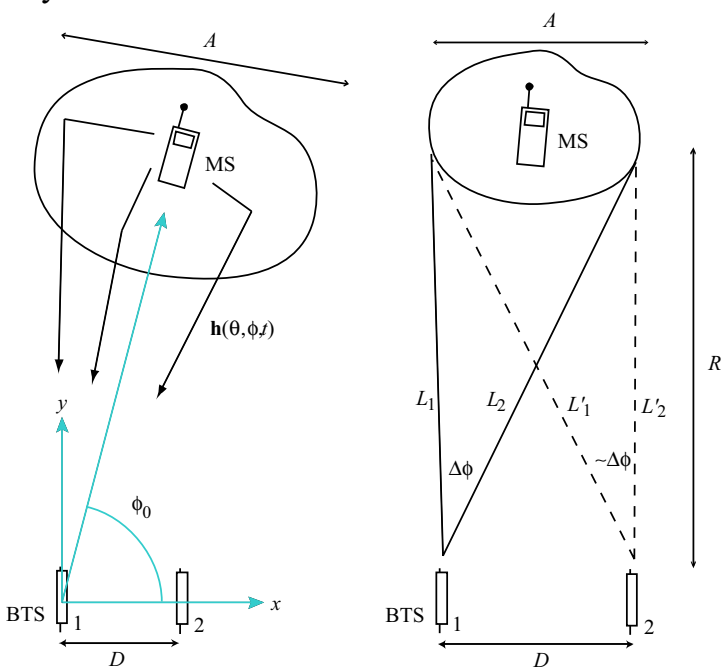

Figure. 10. Geometry of base station diversity where the incident waves on the base station antennas are confined to a sector $\Delta \varphi$ in the horizontal plane.

Figure 10 shows a simplified two-dimensional case of the more general space diversity scenario in Figure 9. A mobile station in a scattering area of size $A$ is transmitting a signal which is scattered and received at antennas 1 and 2 separated by $D$ and at a range $R$. The scattering region $A$ thus occupies an angle of:

$$
\Delta \phi=2 \operatorname{atan} \frac{A / 2}{R} \approx \frac{A}{R}
$$

If we for simplity assume that the incident signals have a uniform angular distribution over $\Delta \varphi$ and that the antennas are identical and vertically polarized with constant pattern over $\Delta \varphi$, the general expression (5) yields

$$
\operatorname{Cov}\left\{\mathrm{V}_{1}, \mathrm{~V}_{2}\right\}=\frac{1}{\Delta \varphi} \frac{\chi}{\chi+1} \int_{\varphi_{0}-\Delta \varphi / 2}^{\varphi_{0}+\Delta \varphi / 2} e^{-j k D \cos \varphi} \partial \varphi
$$

For small $\Delta \varphi$ and normal incidence the correlation reduces to:

$$
\rho=\left|\rho_{c}\right|^{2}=\left(\frac{\sin (2 \pi D \Delta \varphi / 2 / \lambda)}{2 \pi D \Delta \varphi / 2 / \lambda}\right)^{2}
$$

\subsection{Polarization Diversity}

For polarization diversity, we may assume that the fields are incident for a single angle since the two antenna branches are co-located. Expression (5) then reduces to:

$$
\begin{aligned}
& \operatorname{Cov}\left\{\mathrm{V}_{1}, \mathrm{~V}_{2}\right\}=\operatorname{Tr}\left\{\mathbf{E}_{1} \mathbf{E}_{2}{ }^{H} \frac{1}{\chi+1}\left[\begin{array}{ll}
\chi & 0 \\
0 & 1
\end{array}\right]\right\}= \\
& =\frac{E_{1 \theta} E_{2 \theta} \chi+E_{1 \varphi} E_{2 \varphi}}{\chi+1}
\end{aligned}
$$

Fig. 11 shows the difference in diversity gain at $1 \%$ outage calculated form the eigenvalues of the covariance matrices obtained using (5-11). Note that it is important to use the covariance and not only the correlatio, because the variance, i.e. the branch power, will vary when we change the polarization power ratio $\chi$. As expected a large $\chi$ makes space diversity more advantegeous. If we consider unpolarized fields, $\chi=1, \mathrm{w}$ see that a normalized spacing $D \Delta \varphi / \lambda=1$ is required to make the systems equivalent.

In order to compare the two diversity schemes, the question is which values of $\Delta \varphi$ and $\chi$ are relevant? For the polarization power ratio $\chi$, a value between 0 and $6 \mathrm{~dB}$ seems reasonable (see summary in [16]). For the angular distribution $S$, there has been published standard deviation $\sigma_{a}$ of the power azimuth spectrum, also referred to as azimuth spread. Algans et. al [11] present a c.d.f. of $\sigma_{a}$ estimated from TSUNAMI II measurement data. The median values are between $5^{\circ}$ and $8^{\circ}$ for an urban environment. 


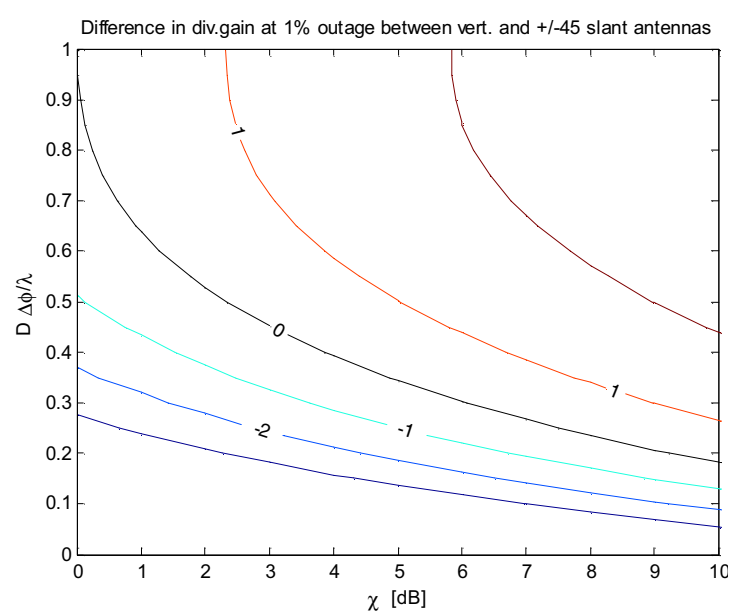

Figure 11. Space vs. polarization diversity calculated form the covariance matrices obtained using(1-3).

The same data was analyzed in [10] and the result was a median between $5^{\circ}$ and $12^{\circ}$. We have in the derivations above assumed a constant distribution. We should then choose the size of the scattering region $\Delta \varphi$ so that the variance is the same as in e.g. the case of a normal distribution. This means that:

$$
\Delta \phi=2 \sqrt{3} \sigma_{a}
$$

Since the use of space diversity can be expected to be limited to sub-urban case, a reasonable value of azimuth spread to consider in our comparisons seem to be $\sigma_{\mathrm{a}} \approx 5^{\circ}$ or 0.1 radian. Thus $\Delta \varphi \approx 0.34$ radians. Hence, an even spacing of $D \approx 3 \lambda$ would make space diversity superior for any $\chi,>1$ !

\section{MIMO SYSTEMS}

In recent years we have seen a great interest in Muliple Input Multiple Output, MIMO, for wireless communication systems. The reason for this is of course the great potential gains that can be achieved from these systems by just adding more antennas at the base station as well as the terminal.

\subsection{Capacity gain}

Example: for a system with $n T$ transmit an $n R$ receive antennas the maximum capacity in bits $/ \mathrm{s} / \mathrm{Hz}$ with no channel information at the transmitter is [12]:

$$
C=\log _{2}\left|\mathbf{I}+\rho \mathbf{H} \mathbf{H}^{H}\right|=\sum_{i=1}^{n_{R}} \log _{2}\left(1+\frac{\rho \lambda_{i}^{2}}{n_{T}}\right)
$$

where $\lambda_{i}^{2}$ are the eigenvalues of $\mathbf{H} \mathbf{H}^{H}$ and $\rho$ is the average SNR at the receive antennas.

If we have LoS, all entries in $\mathbf{H}$ are of magnitude 1 and the rank is 1 . Since we don't have channel information at the transmitter we have to transmit isotropically, i.e. $n_{T}=1$. This means that the eigenvalues are $\left\{\lambda_{1}{ }^{2}, \ldots, \lambda_{i}{ }^{2}, \ldots\right\}=\left\{n_{R}, 0, \ldots, 0\right\}$ and there is only one spatial channel contributing to the capacity and array gain only at the reciever.

If we now increase the number of signal paths, i.e. the rank of without decrease in the $\operatorname{SNR} \rho$, we will increase the capactiy lienarly with $\min \left(n_{R}, n_{T}\right)$. This is seen in Figure 12 where the maximum capacity for $2 \times 2$ and 4 × 4 MIMO systems is plotted vs. SNR.
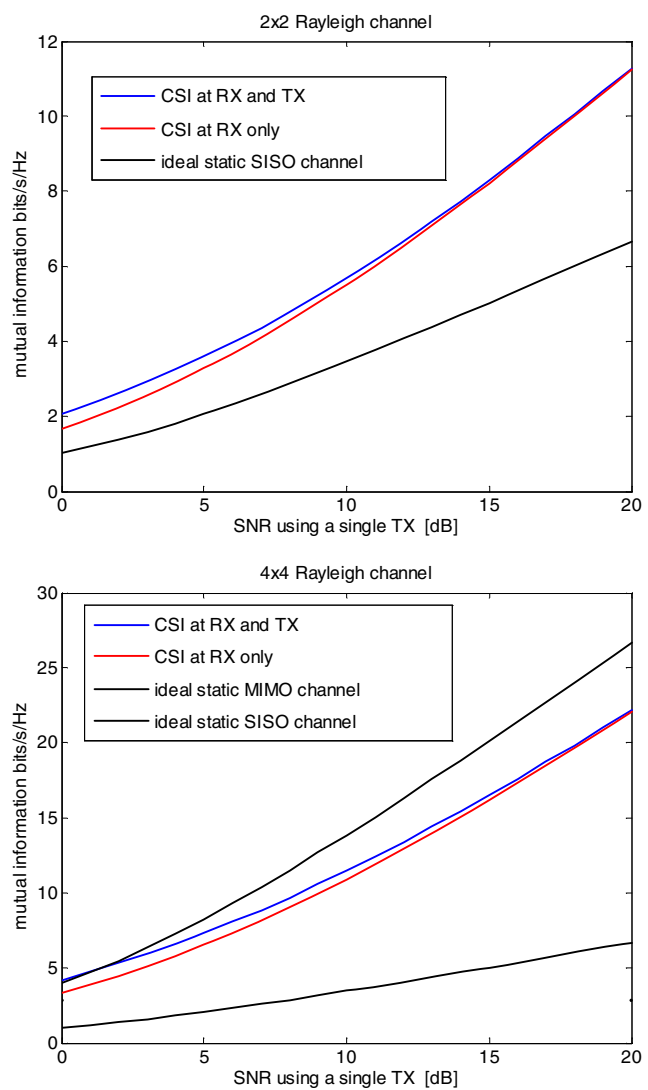

Figure 12. 2x2 (upper) and 4x4 (lower) MIMO capacity vs. SNR with no channel information at the transmitter.

Since multipath propagation is normally associated with increased path loss, we may now ask how much increased loss is acceptable for a MIMO system to be beneficial? If we consider a static Single Input Single Output, SISO, channel with $\mathrm{SNR}=20 \mathrm{~dB}$, we can see that a $2 \times 2$ MIMO system with 8 dB lower SNR may achieve the same capacity. For the 4 x 4 system, we may reduce the SNR by $16 \mathrm{~dB}$.

\subsection{Outdoor to indoor}

Finally, let us look at an example of indoor MIMO capacity with an outdoor base station. Figure. 13 shows the capacity as a function of location for a $4 \mathrm{x}$ 4 MIMO system. As a comparison, a single Rayleigh channel with $10 \mathrm{~dB}$ average SNR provides a maximum capacity of $2.91 \mathrm{bits} / \mathrm{s} / \mathrm{Hz}$. The MIMO 
system allows us to communicate at a significantly higher rate over most of the floor [15].

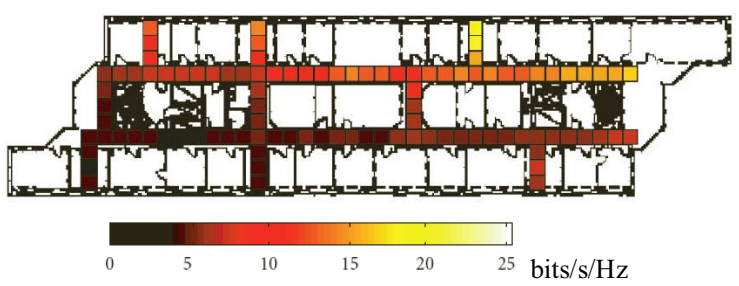

Figure 13. Indoor 4 x 4 MIMO capacity at $1800 \mathrm{MHz}$ with average $\mathrm{SNR}=10 \mathrm{~dB}$ over the whole floor. The base station is positioned in the top right corner [15].

\section{CONCLUSIONS}

The performance of the base station antenna is always a direct result of the requirement of the network it is designed to service. Over the last 20 years we have seen ever changing cellular standards and, hence, changing performance of the base station antennas. Today dual band and dual polarization is standard. But, due to the potential capacity gain, base station antennas for MIMO systems might be as ubiquitous tomorrow!

\section{Acknowledgments}

The authors would like to acknowledge the great contribution to the described development by our coworkers over the years at Allgon and Powerwave

\section{References}

[1] S. Johnsson and D. Karlsson. "Dual band antenna”. USPatent No. 6295028 B1, Sep. 2001.

[2] C.H. Tsao, Y.M Hwang, F. Kilburg, F. Dietrich. "Aperture-coupled patch antennas with widebandwidth and dual-polarization capabilities" IEEE Antennas Propagat. Soc. Symp. Dig., pp. 936 - 939, June 1988.

[3] W. C. Y. Lee and Y. S. Yeh, "Polarization diversity system for mobile radio," IEEE Trans. Com., vol. COM-26, pp. 912-923, Oct. 1972.

[4] Ahlberg, M.; Lindmark, B.; Simons, J.; Beckman, C.;" Downlink propagation measurements in the GSM 900 and $1800 \mathrm{MHz}$ bands", IEEE Antennas Propagat. Soc. Symp. Dig., July 1999, pp. 1506 - 1509

[5] Anderson, S.; Millnert, M.; Viberg, M.; Wahlberg, B. "An adaptive array for mobile communication systems", IEEE Trans. on Veh. Techno. vol. 40, pp. $230-236$, Feb. 1991.

[6] Ekstrom, H.; Furuskar, A.; Karlsson, J.; Meyer, M.; Parkvall, S.; Torsner, J.; Wahlqvist, M.;' Technical solutions for the $3 \mathrm{G}$ long-term evolution", IEEE Com. Mag., vol. 44, pp. 38 45, 2006.

[7] W. C. Y. Lee, Mobile cellular telecommunication, McGraw-Hill New York, 1995.

[8] C. A. Balanis. Antenna Theory - Analysis and Design, $2^{\text {nd }}$ Edition, John Wiley \& Sons, 1997.

[9] B. Lindmark, S. Lundgren, J.R. Sanford, and C. Beckman. "Dual-polarized array for signalprocessing applications in wireless communications" IEEE Trans. Antennas Propagat., vol. 46, pp. 758 - 763, June 1998.

[10]K.I Pedersen, P.E Mogensen, B.H. Fleury, A stochastic model of the temporal and azimuthal dispersion seen at the base station in outdoor propagation environments, IEEE Trans. Veh. Technol., vol. 49, pp. 437 - 447, March 2000.

[11]A. Algans, K.I. Pedersen, P.E. Mogensen, "Experimental analysis of the joint statistical properties of azimuth spread, delay spread, and shadow fading", IEEE Journal Sel. Areas Comm., Vol. 20, pp. 523 - 531, April 2002.

[12]G. J. Foschini and M. J. Gans, "On limits of wireless communications in a fading environment when using multiple antennas". IEEE Personal Comm., Vol. 6, pp 311-335, March 1998.

[13]R.E. Collin and F. Zucker, Antenna Theory, Part 1, New York: McGraw-Hill, 1969.

[14]R. Vaughan and J. Bach Andersson, "Antenna Diversity in Mobile Communications", IEEE Trans. Veh. Comm., vol. VT-36, Nov. 1987.

[15]L. Garcia-Garcia, B. Lindmark, N. Jaldén, P. Zetterberg, and L. de Haro, "Measurements of MIMO Indoor Channels at $1800 \mathrm{MHz}$ with Multiple Indoor and Outdoor Base Stations", EURASIP Journal on Wireless Communications and Networking, in press, 2007.

[16]B. Lindmark, and M. Nilsson, M. Dept. of Electromagnetics. "On the available diversity gain from different dual-polarized antennas" IEEE Journal Sel. Areas Comm., vol. 19, pp: $287-294$, Feb 2001. 